\title{
The Effect of Photodynamic Therapy in the Treatment of Chronic Periodontitis: A Review of Literature
}

\author{
Mansour Meimandi', Mohammad Reza Talebi Ardakani', Azadeh Esmaeil Nejad ${ }^{1 *}$, Parisa Yousefnejad ${ }^{2}$, Khosro \\ Saebi $^{2}$, Mohammad Hossein Tayeed ${ }^{3}$
}

'Periodontics Department, School of Dentistry, Shahid Beheshti University of Medical Sciences, Tehran, Iran

${ }^{2}$ Private Practice, Tehran, Iran

${ }^{3}$ Dental Student, School of Dentistry, Shahid Beheshti University of Medical Sciences, Tehran, Iran

\author{
*Correspondence to \\ Azadeh Esmaeil Nejad, D.D.S, \\ MSc; Assistant Professor, \\ Periodontics Department, School \\ of Dentistry, Shahid Beheshti \\ University of Medical Sciences, \\ Tehran, Iran. \\ Tel: +98 9123704781; \\ Fax: +98 2122403194; \\ Email: \\ esmaeilnejada@yahoo.com
}

Published online 29 August 2017

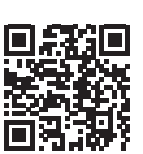

\begin{abstract}
Introduction: Chronic periodontitis is the most common periodontal disease which is related to the chronic accumulation of bacterial plaque. Since mechanical methods are not sufficient in the treatment of this disease, administration of local/systemic antibiotic is recommended following mechanical debridement. However, side effects of antibiotics such as microbial resistance and patient allergy led to development of alternative methods. One of these suggested methods is the antimicrobial photodynamic therapy (aPDT). PDT is a local noninvasive treatment modality without the side effects caused by antibiotics. The aim of this study was to review the articles related to the application of PDT with laser in the treatment of chronic periodontitis.

Review of literature: In the present review of literature, the authors used key words such as chronic periodontitis, laser and photodynamic therapy, and conducted a literature search via Google Scholar and PubMed for the period of 1990 to 2015. A total of 47 articles in English were found. The articles that were not associated with the topic of research and review articles were deleted and only clinical trials were evaluated. After reviewing 23 articles' abstracts, the full texts of 16 articles were analyzed.

Conclusion: Considering the safety, the lack of side effects and general advantages like more patient compliance, the PDT treatment with scaling and root planing (SRP) is recommended as an efficient adjunctive modality for the treatment of localized chronic periodontitis especially during the maintenance phase in non-surgical treatment.

Keywords: Chronic periodontitis; Laser; Photodynamic therapy.
\end{abstract}

\section{Introduction}

Chronic periodontitis is one of the most common periodontal diseases and bacterial biofilm is its etiologic factor. ${ }^{1}$ Of the most important pathogens causing periodontal disease, complex microorganisms such as Aggregatibacter Actinomycetemcomitans, Porphyromonas gingivalis, and Treponema denticola, Tannerella forsythia bacteria can be noted. ${ }^{2}$

Due to the fact that the main goal of periodontal treatments is to reduce microbial agents, common treatments such as the mechanical debridement (the use of ultrasonic equipment and manually) as well as the use of topical or systemic antibiotics are considered as the main therapeutic measures in the treatment of chronic periodontitis. ${ }^{3}$ Although the use of antibiotics can reduce the periodontal pathogens, their frequent use can cause bacterial resistance. Also, causing allergy in patients and the inability to make proper concentration of drug in the periodontal pockets are other disadvantages of using antibiotics. $^{4}$
In addition, in a systematic review, some of antibiotics' side effects such as skin rash, itching, oral candidiasis, nausea, vomiting, have been proposed.

Moreover, due to the complex anatomy of the furca area, the pockets depth, and the penetration of microorganisms into tissues, it is difficult to have an appropriate access to clean up. Therefore, for the reasons outlined, efforts to find adjunctive treatment have increased. ${ }^{6}$ One of these treatments is photodynamic therapy (PDT).

This method was first used in 1990 for the treatment of cancer. It was determined that its use stimulates autophagy (a method of cell catabolism, which leads to the destruction of abnormal cells) in resistant cancer cells or precancerous cells. In this method, wavelengths between $650-900 \mathrm{~nm}$ which are within the visible red light and near infrared, and have great influence on biological tissue are used. So far, more than 400 substances have been identified as photosensitizers, including Indocyanine Green. After excitation with light, this substance gains properties such as wound healing, antibacterial effect and

Please cite this article as follows: Meimandi M, Talebi Ardakani MR, Esmaeil Nejad A, Yousefnejad P, Saebi K, Tayeed MH. The effect of photodynamic therapy in the treatment of chronic periodontitis: a review of literature. J Lasers Med Sci. 2017;8(Suppl 1):S7-S11. doi:10.15171/jlms.2017.s2. 
the treatment of chronic skin and mucosa infections. This substance is very safe, does not cause any harm to the host cells and it has been approved by the Food and Drug Administration (FDA) ${ }^{7,8}$ According to Allison et al, PDT involves the use of 3 components: (1) Light, (2) Oxygen free radicals, and (3) Photosensitizer. ${ }^{9}$

When the photosensitizer is stimulated by an appropriate light wavelength (wavelengths between 650-900 nm which are within the visible red light and near infrared), it provides free radicals of oxygen that causes tissue damage $\left.{ }^{10,11}\right)$. The cytotoxic products have a short halflife (about $0.04 \mu \mathrm{s})$ and limited radius effect $(0.20 \mu \mathrm{m})$. In other words, they are limited to the infected area, where the photosensitizer is accumulated. Thus, PDT is a topical method that does not affect other host tissues. ${ }^{12}$

Due to its safety, no side effects and more acceptability of non-surgical methods of treatment to patients, PDT is important as an adjunctive therapeutic method with scaling and root planing (SRP) in order to increase the efficiency of non-surgical treatments.

Therefore, the aim of this study was to evaluate the effects of PDT using laser in the treatment of chronic periodontitis. Thus, in this review of literature, we examined the medical literature, seeking to identify and synthesize relevant information to formulate the best approach to treat chronic periodontitis.

\section{Review of Literature}

In the present review of literature, the authors used key words such as laser, chronic periodontitis, and photodynamic therapy, and conducted a literature search via Google Scholar and PubMed for the period of 1990 to 2015. A total of 47 articles in English were found. The articles that were not associated with the topic of research and review articles were deleted and only clinical trials were evaluated. After reviewing 23 articles' abstracts, the full texts of 16 articles were analyzed.

In a randomized clinical trial that was conducted by Andersen et al in 2007, 23 patients with chronic periodontitis were randomly divided into 3 groups: group 1- treatment with PDT, group 2- treatment with SRP and PDT (laser diodes) and group 3- treatment with SRP alone (control group). The results of the study showed that the addition of PDT to SRP statistically significantly improved the clinical attachment level (CAL) and the depth of probing pocket (PPD). ${ }^{13}$

In another randomized clinical trial that was conducted by Braun et al in 2008, the main purpose was to compare clinical outcomes of doing SRP with or without PDT. In this study, 20 patients with chronic periodontitis were randomly divided into 2 groups by using the split-mouth method. All patients were under SRP. In a quadrant, PDT was performed by means of laser diode $660 \mathrm{~mm}$ and photosensitizer phenothiazine chloride.

At the beginning of the study, 1 week and 3 months after treatment, the gingival crevicular fluid (GCF), PPD, bleeding on probing (BOP)and gingival recession (GR) were measured. In all cases except for GR, better results were obtained in the group treated with PDT; however, there was no difference in the recession in the 2 groups. ${ }^{14}$ In another clinical trial that was conducted in 2008 by Christodoulides et al, 24 patients with chronic periodontitis were randomly divided into 2 groups: 1treatment with SRP, 2-treatment with SRP and PDT (diode laser $670 \mathrm{~nm}$ and power of $75 \mathrm{~mW}$ ) and no statistically significant difference was seen between the 2 groups, and only BOP showed significant improvements in the group treated with SRP and PDT, compared to the one treated with SRP alone. ${ }^{15}$

In another clinical trial conducted in 2009 by Chondros et al, 24 patients with chronic periodontitis were divided into 2 groups: group 1- treatment by SRP and group 2- treatment with SRP and PDT $(670 \mathrm{~nm}, 75 \mathrm{~mW}$ laser diode and photosensitizer phenothiazine chloride for 60 seconds), respectively. Both groups were assessed at 2 time periods of 3 and 6 months. No significant differences in terms of PPD, CAL, and the amount of plaque were seen, but the BOP was significantly reduced in the group of treatment by SRP and PDT. ${ }^{16}$

In 2009, Polansky et al, treated 58 patients with chronic periodontitis in 2 treatment groups: 1-treatment with SRP and 2- treatment with SRP and PDT (laser diode 680 and $75 \mathrm{~mW}$ ), and no difference in the amount of CAL, BOP, and PPD were seen between the 2 groups. Based on the results obtained, the use of PDT once, did not have significant clinical effect compared to SRP treatment alone. ${ }^{17}$ In a similar study done by Ruhling et al on 25 patients using $636 \mathrm{~nm}$ diode laser photo synthesizer phenothiazine chloride, similar results were obtained. ${ }^{18}$

Lui et al in 2011 in a clinical trial investigated 24 patients with chronic periodontitis. The patients were randomly divided into 2 groups: group 1- under SRP treatment and group 2-under SRP and PDT treatment (diode laser $940 \mathrm{~nm}$ for 3 days). A month later, BOP and GCF had a greater reduction in the first group, but after 3 months no difference between the 2 groups was seen. Also, the amount of IL- $1 \beta$ had dropped more in the second group than the control group in a week; therefore, the researchers concluded that the use of PDT with SRP is more effective in improving patients' periodontal health in short term. ${ }^{19}$ In 2011, Aykol et al divided 24 patients with chronic periodontitis into two groups during a clinical trial. Group 1 was treated with SRP, and group 2 was treated with SRP and PDT (diode laser $808 \mathrm{~nm}$, power of $0.25 \mathrm{~W}$ ) on the first, second and seventh day of treatment. Then all the patients were divided into 2 groups of smokers and non-smokers. The group treated with SRP and PDT for both smokers and non-smokers showed better results in terms of improved clinical parameters. ${ }^{20}$

In 2011, Cappuyns et al randomly assigned 32 patients with a history of non-surgical chronic periodontitis treatment into 3 groups in a clinical trial: (1) treatment with SRP, (2) treatment with diode laser $810 \mathrm{~nm}$, and (3) treatment with PDT, diode laser $660 \mathrm{~nm}$ and photosensitizer phenothiazine chloride. All of the 3 groups showed an improvement in GR, BOP and PPD clinical parameters 
and there was not any difference between them. ${ }^{21}$

In 2012, Noro Filho et al investigated 12 patients with HIV and chronic periodontitis treatment history in a clinical trial by split-mouth method. They have randomly divided them into 2 groups: (1) treatment with SRP and (2) treatment with SRP and PDT (diode laser $660 \mathrm{~nm}$, and photosensitizer methylene blue). Six months later they have observed improvements in PPD and CAL clinical parameters in the second group. ${ }^{22}$

In another clinical trial investigation in 2012, Berakdar et al randomly assigned 22 patients with chronic periodontitis with at least 4 teeth in each quadrant with residual pockets over $5 \mathrm{~mm}$ into 2 groups: (1) treatment with SRP and (2) treatment with SRP and PDT (laser 670 $\mathrm{nm}, 150 \mathrm{~mW}$ and photo sensitizer $0.005 \%$ methylene blue. They have observed PD, CAL and BOP improvement in both groups but the amount of PD improvement was significant in the second group. ${ }^{23}$

In a study in 2012, Giannelli et al compared the clinical outcomes of chronic periodontitis treatment with SRP and SRP combined with PDT and photo-ablation with diode laser. They have perused 26 patients. The results of the study showed that treatment of chronic periodontitis with photo-ablation along several PDT sessions is more effective in improving patients' periodontal health condition compared to SRP alone. ${ }^{24}$

In 2013, during a clinical trial, Balata et al have divided 22 patients presenting chronic periodontitis with depth of 5-7 mm pockets in each quadrant into 2 groups, using split mouth method. Group 1 was treated with SRP. Group 2 was treated with SRP and PDT (laser $660 \mathrm{~nm}$, $100 \mathrm{~W}$ and photo sensitizer methylene blue $0.005 \%$ ). They have checked BOP, CAL and PD clinical parameters before the treatments as well as 1 month, 3 months and 6 months after the treatment. In each group no statistically significant difference was observed clinically after the treatment. ${ }^{25}$

In 2013, Souza et al studied the impacts of microbial PDT on the level of transforming growth factor (TGF) $\beta_{1}$ in GCF on patients. In that study they chose 50 patients with bilateral grade III furcation involvement in mandibular molars. Each pair of molar teeth was randomly divided into experimental group and control group. They have applied SRP into control group and SRP accompanied with PDT applied into the experimental group. Forty-five days later, the control group had received flap surgery and SRP, and the experiment group had received flap surgery, SRP and PDT. They have checked GCF collected by ELISA method by the amount of TGF $\beta_{1}$ at baseline, 45 days after the initial treatment and 21 days after the surgical treatment. The experiment group compared to the control group at 45 days after the initial treatment and 21 days after the surgical treatment has shown statistically significant differences. ${ }^{26}$

In 2014, Pourabbas et al carried out a clinical trial for 3 months on 22 patients affected with chronic periodontitis, which has shown that adding one extra therapy session with toluidine blue and concentrated light of $638 \mathrm{~nm}$ does not add any extra improvement on clinical indicators. Although that would causes reducing the rate of tumor necrosis factor alpha (TNF- $\alpha$ ) in gingival sulcus fluid, flashing concentrated light only once along scaling may impact the ability to show the effects of treatment with PDT, and makes it impossible to interpret the results. ${ }^{27}$

In 2014, Kolbe et al randomly assigned 22 patients with chronic periodontitis during a clinical trial by split mouth method. They have divided the patients into 3 groups based on the random points they have been selected before: (1) Treatment with SRP, (2) Treatment with SRP and photosensitizer, and (3) Treatment with SRP and PDT. Patients were checked at baseline, 3 months and 6 months after it was done. The results of the study showed that each group resulted in improvement of clinical conditions but only in the third group, an increasing of IL- 4 perception and reduction of IL- 6, IL- 8 and IL- $1 \beta$ were reported. ${ }^{28}$

\section{Discussion}

The aim of this study was to evaluate the effects of PDT using laser in periodontitis treatment. The results of studies done on the impact of PDT as an adjunctive way of treatment for chronic periodontitis are conflicting. Although in most of these studies, laser diode has been used, but none of them mentioned which types of laser, photosensitizer, wavelength, power and intensity are more effective. ${ }^{29}$

It appears that the use of multiple doses of laser is more effective than only one dose. ${ }^{30}$ But only 1 of the 5 studies shows that using laser results positively in improving clinical parameters. In a study carried out by Malik et al in 2010, they concluded that only one use of laser does not affect patients with chronic periodontitis. Laser therapy should be repeated several times in the first week of treatment to reveal the antimicrobial effects. ${ }^{31}$

Although the use of laser in many studies showed similar results to SRP, but we cannot disregard the shorter treatment time, more rapid onset of action, reduced need for anesthesia and the absence of bacterial resistance occurrence when using laser.

It seems that the most useful effect in using laser is in the maintenance phase because usually at this time retreatment is limited to residual deep pockets and is localized. Using laser prevents removing extra dental tissues. Also this method prevents tooth sensitivity.

In addition, there is no need of using antibiotics after surgery due to the use of laser with its anti-bacterial effect. Since the use of PDT reduces bacteremia, it may be recommended for patients who are at risks of endocarditis. The use of PDT as an adjunct therapy in non-surgical treatments is economical in comparison to periodontal surgeries.

In addition, due to its focal effect, using systematic antibiotics is unnecessary. Also high concentrated photosensitizer at the site of infection plays an important role in reducing the bacteria without adverse effects on host cells. ${ }^{32}$ 
Overall, with general attention to the safety, other benefits and no side effects, PDT is recommended as an adjunctive therapy with SRP for the treatment of localized periodontitis, especially during the maintenance phase, to complete and increase the efficiency in non-surgical treatments.

Considering the fact that the important role of laser in the treatment of periodontal diseases and in reducing the periodontal pathogens such as A. actinomycetemcomitans and $P$. gingivalis has been completed and approved, comparing different variety of photosensitizer, laser powers and wavelength would bring the best and the most effective clinical outcomes. Also the frequency of laser sessions, the duration, and other laser properties should be evaluated to determine the best way to use it.

\section{Conclusion}

Of the 16 articles studied, in 8 of them using PDT with SRP caused improvement in CAL, PPD and BOP (clinical parameters), and in the rest of them using PDT with SRP together did not result in any improvement. But in most of them PDT and SRP caused significant improvement in BOP compare to SRP alone. Also using PDT several times is more effective than only once.

\section{Ethical Considerations}

Not applicable.

\section{Conflict of Interests \\ None.}

\section{References}

1. Bassir SH, Moslemi N, Jamali R, et al. Photoactivated disinfection using light-emitting diodes as an adjunct in the management of chronic periodontitis:a pilot double-blind split- mouth randomized clinical trial. J Clin Periodontol. 2013;40(1):65-72. doi:10.1111/jcpe.12024.

2. Tanner AC. Anaerobic culture to detect periodontal and caries pathogens. J Oral Biosci. 2015;57(1):18-26. doi:10.1016/j.job.2014.08.001

3. Cobb CM. Non-surgical pocket therapy: mechanical. Ann Periodontol. 1996;1(1):443-490. doi:10.1902/ annals.1996.1.1.443.

4. Slots J, Rams TE. Antibiotics in periodontal therapy:advantages and disadvantages. J Clin Periodontol. 1990;17:479-493.

5. Gillies M, Ranakusuma A, Hoffmann T, et al. Common harms from amoxicillin: a systematic review and metaanalysis of randomized placebo-controlled trials for any indication. CMAJ. 2015;187(1):E21-E31. doi: 10.1503/ cmaj.140848.

6. Petersilka GJ, Tunkel J, Barakos K, Heinecke A, Häberlein I, Flemmig TF. Subgingival plaque removal at interdental sites using a low-abrasive air polishing powder. J Periodontol. 2003;74:307-311.

7. Parker S. The use of diffuse laser photonic energy and indocyanine green photosensitizer az an adjunct to periodontal thrrapy. Br Dent J. 2013;215(4):167-171. doi:10.1038/sj.bdj.2013.790.

8. Garg AD, Maes H, Romano E, Agostinis P. Autophagy a major adaptation pathway shaping cancer cell death and anti ancer immunity responses following photodynamic therapy. Photochem Photobiol Sci. 2015;14:1410-1424.

9. Allison RR, Baganto VS, Cuenca R, Dowinie GH, Sibata $\mathrm{CH}$. The future of photodynamic therapy in oncology. Future Oncol. 2006;2:53-71.

10. Raghavendra M, Koregol A, Bhola S. Photodynamic therapy: a targeted therapy in eriodontics. Aust Dent J. 2009;54:S102-S109. doi:10.1111/j.1834-7819.2009.01148.x.

11. Ochsner M. Photophysical and photobiological processes in the photodynamic therapy of tumours. J Photochem Photobiol B. 1997;39:1-18.

12. Moan J, Berg K. The photodegradation of por hyrins in cell that can be used to estimate the lifetime of singlet oxygen. Photochem Photobiol. 1991;53:549-553.

13. Andersen R, Loebel N, Hammond D, Wilson M. Treatment of periodontal disease by photodisinfection compared to scaling and root planing. J Clin Dent. 2007;18(2):34-38.

14. Braun A, Dehn C, Krause F, Jepsen S. Short-term clinical effects of adjuctive antimicrobial photodynamic therapy in periodontal treatment:a randomized clinical trial. $J$ Clin Periodontol. 2008;35(10):877-84. doi:10.1111/j.1600051X.2008.01303.x.

15. Christodoulides N, Nikoldakis D, Chondros $P$, et al. Photodynamic therapy as an adjunct to non-surgical periodontal treatment a randomized controlled clinical trial. J Periodontol. 2008;79(9):1638-44. doi:10.1902/ jop.2008.070652

16. Chondros P, Nikolidakis D, Christodoulides N, Rossler $\mathrm{R}$, Gutknecht N, Sculean A. A photodynamic therapy as adjunct to non-surgical periodontal treatment in patients on periodontal maintenance: a randomized, controlled clinical trial. Laser Med Sci. 2009;24:681-688.

17. Polansky R, Haas M, Heschl A, Wimmer G. Clinical effectiveness of photodynamic therapy in treatment of periodontitis. J Clin Periodontol. 2009;36:575-580.

18. Ruhling A, Fanghanel J, Hushmand M, et al. Photodynamic therapy of persistent pockets in maintenance patients - a clinical study. Clin Oral Investig. 2010;14(6):637-644. doi:10.1007/s00784-009-0347-4.

19. Lui J, Corbet EF, Jin L. Combined photodynamic and low -level laser therapies as an adjunct to non-surgical treatment of chronic periodontitis. J Periodontol Res. 2011;46:89-96.

20. Aykol G, Baser U, Maden I, et al. The effect of low- level laser therapy as an adjunct to non-surgical periodontal treatment. J Periodontol. 2011;82(3):481-488. doi:10.1902/ jop.2010.100195.

21. Cappuyns I, Cionea N, Wick P, Giannopoulou C, Mombelli A. Treatment of residual pockets with photodynamic therapy, diode laser, or deep scaling. A randomized split- mouth controlled clinical trial. Lasers Med Sci. 2012;27(5):979-986. doi:10.1007/s10103-011-1027-6.

22. Noro Filho GA, Casarin RC, Casti MZ, Giovani EM. PDT in non- surgical treatment of periodontitis in HIV patients:a split-mouth, randomized clinical trial. Lasers Surg Med. 2012;44(4):296-302. doi:10.1002/lsm.22016.

23. Berakdar M, Callaway A, Fakhrddin M, Rob A, Willershausen B. Comparison between scaling-root planning and SRP/photodynamic therapy: six-month study. Head Face Med. 2012;8:12.

24. Giannelli M, Formigli L, Lorenzini L, Bani D. Combined photoablative and photodynamic diode laser therapy as an adjunct to non-surgical periodontal treatment: 
a randomized split-mouth clinical trial. $J$ Clin Periodontol. 2012;39(12):962-970. doi:10.1111/j.1600051X.2012.01925.x.

25. Balata ML, Andrade IP, Santos DB, et al. Photodynamic therapy associated with full-mouth ultrasonic debridement in the treatment of severe chronic periodontitis:a randomized-controlled clinical trial. J Appl Oral Sci. 2013;21(12):208-214. doi:10.1590/1678-7757201302366.

26. Souza sl, Andrade PF, Silva JS, et al. Effect of antimicrobial photodynamic therapy on transforming growth factor- B1 levels in the gingival crevicular fluid. Photomed Laser Surg. 2013;31:65-71.

27. Pourabbas R, Kashefimehr A, Rahmanpour N, et al. A Effects of photodynamic therapy on clinical and gingival crevicular fluid inflammatory biomarkers in chronic periodntitis: a split-mouth randomized clinical trial. J Periodontol. 2014;85(9):1222-1229. doi:10.1902/ jop.2014.130464.
28. Kolbe MF, Fernanda V, Ribeir O, et al. Photodynamic therapy during supportive periodontal care: clinical microbiologic immunoinflammatory, and patient-centered performance in a split-mouth randomized clinical trial. J Periodontol. 2014;85(8):e277-e286. doi:10.1902/ jop.2014.130559.

29. Chan Y, Lai CH. Bacteriocidal effects of different laser wavelengths on periodontopathic germs in photodynamic therapy. Lasers Med Sci. 2003;18:51-55.

30. de Paula Eduardo C, de Freitas PM, Esteves-Oliveira M, et al. Laser phototherapy in the treatment of periodontal disease. Lasers Med Sci. 2010;25(6):781-792. doi: 10.1007/ s10103-010-0812-y.

31. Malik R, Manocha A, Suresh DK. Photodynamic therapy: a strategic review. Indian J Dent Res. 2010;21:285-291.

32. Takasaki AA, Aoki A, Mizutani K, et al. Application of antimicrobial photodynamic therapy in periodontal and peri - implant diseases. Periodontol 2000. 2009;51:109-140. 